\title{
Unmanned Aerial Vehicle (UAV) Dynamic-Tracking Directional Wireless Antennas for Low Powered Applications That Require Reliable Extended Range Operations in Time Critical Scenarios
}

Matthew O. Anderson Scott G. Bauer James R. Hanneman

October 2005

The INL is a U.S. Department of Energy National Laboratory operated by Battelle Energy Alliance

Idaho National Laboratory 
INL/EXT-05-00883

\section{Unmanned Aerial Vehicle (UAV) Dynamic-Tracking Directional Wireless Antennas for Low Powered Applications That Require Reliable Extended Range Operations in Time Critical Scenarios}

Matthew G. Anderson

Scott G. Bauer

James R. Hanneman

October 2005

Idaho National Laboratory

Idaho Falls, Idaho 83415

Prepared for the

U.S. Department of Energy

Office of Nuclear Energy

Under DOE Idaho Operations Office

Contract DE-AC07-05ID14517 


\title{
Unmanned Aerial Vehicle (UAV) \\ Dynamic-Tracking Directional Wireless Antennas for Low Powered Applications that Require Reliable Extended Range Operations in Time Critical Scenarios
}

\author{
Matthew O. Anderson, Scott G. Bauer, James R. Hanneman \\ Idaho National Laboratory, 2525 Fremont Avenue, Idaho Falls, Idaho,83415, scott.bauer@inl.gov, \\ matthew.anderson@inl.gov,james.hanneman@inl.gov
}

\begin{abstract}
The proven value of DOD Unmanned Aerial Vehicles (UAVS) will ultimately transition to National and Homeland Security missions that require real-time aerial surveillance, situation awareness, force protection, and sensor placement. Public services first responders who routinely risk personal safety to assess and report a situation for emergency actions will likely be the first to benefit from these new unmanned technologies. 'Packable' or 'Portable' small class UAVS will be particularly useful to the first responder. They require the least amount of training, no fixed infrastructure, and are capable of being launched and recovered from the point of emergency.
\end{abstract}

All UAVs require wireless communication technologies for real- time applications. Typically on a small UAV, a low bandwidth telemetry link is required for command and control $\left(C^{2}\right)$, and systems health monitoring. If the UAV is equipped with a real-time Electro-Optical or Infrared (EO/Ir) video camera payload, a dedicated high bandwidth analog/digital link is usually required for reliable high-resolution imagery. In most cases, both the wireless telemetry and real-time video links will be integrated into the UAV with unity gain omni-directional antennas. With limited on-board power and payload capacity, a small UAV will be limited with the amount of radio-frequency $(R F)$ energy it transmits to the users. Therefore, 'packable' and 'portable' UAVs will have limited useful operational ranges for first responders.

This paper will discuss the limitations of small UAV wireless communications. The discussion will present an approach of utilizing a dynamic ground based real-time tracking high gain directional antenna to provide extend range stand-off operation, potential RF channel reuse, and assured telemetry and data communications from low-powered UAV deployed wireless assets.

\section{INTRODUCTION}

Unmanned Aerial Vehicles (UAV) systems are making the transition from military applications to National and Homeland Security emergency response scenarios. In many situations UAVs have the ability to quickly assess time-sensitive events, and disseminate dedicated situational knowledge for decision makers and response crews. Maturing small UAVs are well suited for emergency response. They require limited operator skills, don't need complex manned aviation support and infrastructure, and don't risk a manned operator. UAV systems will only mature in capability as more are deployed and utilized.

UAVs can be categorized in several classes or sizes. Two such classes of UAVs well suited for emergency response can be described as 'packable' and/or 'portable'. 'Packable' UAVs have the ability to be hand carried and deployed and 'portable' UAVs require limited vehicle and trailer support for field deployment. Each has advantages, and like all aviation tools, one size seldom fits all. It should be noted that all UAVs are an engineered integrated system of systems that include propulsion, aeromechanical, avionics, power, mission planning, safety and health, sensors, wireless communications, and payload systems. Special attention to details and management of risk can mean the difference between success and failure.

The Idaho National Laboratory (INL) is engaged in the Development, Test, and Evaluation (DT\&E) of 'portable' UAV technologies for first responder safeguards and security applications [Fig. 1]. An airframe and subsystems have been integrated and deployed in a variety of configurations with maturing missions for service with security first responders. It's a program shared jointly between the engineers developing the UAV systems, and the safeguards and security personnel who are discovering best methods for technology service. A driving factor in all technologies developed on the program is best use of affordable technology.

One of the most critical subsystems to our First Responder UAV is the wireless communications links for 


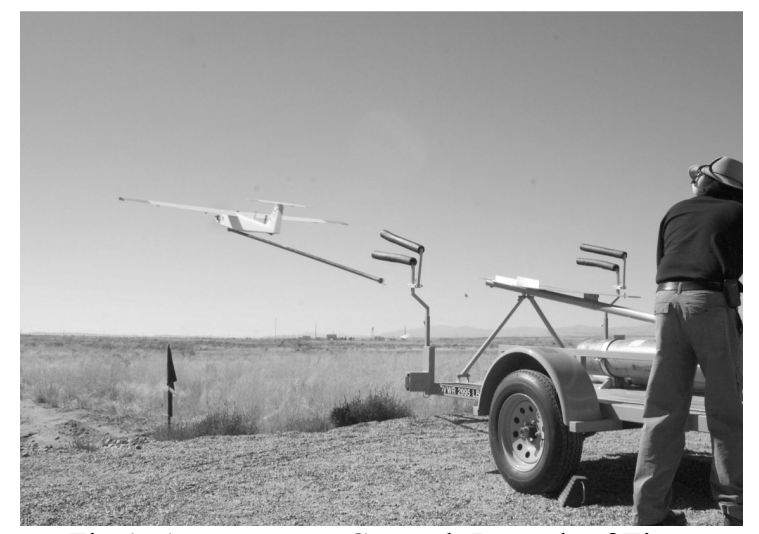

Fig 1. Autonomous Catapult Launch of First Responder UAV

both telemetry and data. Without robust and reliable wireless communications, the UAV will at a minimum be unsafe to operate and provide emergency responders with added technology distractions versus the collection of time-sensitive information. Because of size and power limitations on-board 'portable' UAVs, creative wireless solutions that increase range and reliability are required that don't add additional weight, complexity, and power consumption to the UAV.

As part of INL's First Responder UAV DT\&E project, the INL has analyzed, designed, prototyped, integrated, and tested a dynamic tracking directional antenna to support high gain robust communications from a static ground station to a dynamic airborne UAV possessing a small omni-direction antenna. The following paper will highlight this simple and affordable method to increase the quality and reliability of a communications link to a small UAV without adding additional equipment to the UAV or substantial cost to the system.

It should be acknowledged that wireless directional communications has long been used with other technologies including configurations with other UAVs. Fundamentally, this is another applied approach that when employed could create substantial gains in assured communications at extended ranges to the user of remote unmanned systems.

\section{BASELINE WIRELESS UAV CONFIGURATION}

The effort addressed in this paper will focus on extending the range and reliability of the UAV wireless bi-directional telemetry link used for command and control of the UAV. A dynamic tracking directional antenna will be applied to the bi-directional communication link and performance will be characterized. With appropriate engineering, the same type of configuration and analysis could be applied to other communications links including the wireless video transmitter for real-time EO/IR video for the UAV. In practical terms, there are other systems in similar configurations that would benefit from the same type of dynamic tracking directional antenna, not just UAVs.

The INL First Responder UAV is similar in construction to other unmanned systems in its class. It currently utilizes a Piccolo Plus avionics package from Cloud Cap Technology (www.cloudcaptech.com). The autonomous autopilot and ground station includes an embedded OEM MHX-910 spread spectrum wireless communications data link from Mircohard Systems (www.microhardcorp.com). Standard configuration for the autopilot includes $1 / 4$ wave 'rubber duck' style $2 \mathrm{dBi}$ gain omni-directional antenna, and the ground station includes a $5 \mathrm{dBi}$ gain omni-directional 'wip' style antenna. Both are typical well-matched non-optimized low-cost off the shelf solutions one could expect to see with a small UAV. Both in theory require installations with 'good' ground planes for optimal electrical performance. In our real-world installation flown at the INL, the antenna performance appears less than what should be achievable from the antenna specifications.

Command and Control of the UAV is accomplished real-time through the wireless communications link via a Windows ${ }^{\mathrm{TM}}$ based application and laptop connected to the ground station. During UAV in flight operations the autonomous avionics continuously transmits a $1 \mathrm{~Hz}$ telemetry stream that includes vital information on UAV performance, health, and status. Most importantly for this effort, the telemetry stream transmitted to the ground station includes Global Position System (GPS) coordinates in latitude, longitude, elevation, and in addition barometric altitude is reported.

As with many wireless communication systems, actual performance will vary with interfering sources at the time the system is deployed in the field. These interfering sources, such as signal reflections, refraction, or non line of sight conditions (NLOS), could cause propagation loss and performance degradation. Furthermore an antenna will collect energy from competing energy sources, reducing the signal to noise level and challenging the sensitivity of the receiver. All these factors come into play during UAV fielding and will limit the theoretical performance of the bi-directional communications link. In most cases the environment is something the emergency responders need to accept. In the configuration described above at the INL UAV Test Facilities, we will typically experience acceptable real time communications for safe operations up to $8-10 \mathrm{~km}$ line of sight. During deployments at different locations in the U.S. we have seen operational ranges of more and less than those achieved at the INL.

\section{RF PROPOGATION ANALYSIS AND ANTENNA SELECTION}

Directional high gain antennas are obvious choices for many wireless communications configurations where 
performance is being optimized based upon point to point RF systems designs. In most cases they are inexpensive and readily available in many configurations. Using a directional antenna allows energy to be directed and received from a known location, potentially minimizing the negative effects of competing energy sources outside the antenna pattern.

Radio Frequency propagation analysis and modeling was completed using HTz Warfare by ATDI (www.atdi.com). The software supports simulation of point to multipoint communications systems such as cellular, mobile radios, IEEE 802.xx, satellite communications, microwave, and electronic warfare (EW) applications. The electromagnetic propagation results can be analyzed to determine link reliability, system coverage, and potential interference concerns. The current use of the software at the INL is to accurately modeling the coverage area of the Wireless Test Bed infrastructure under various test conditions for National and Homeland Security applications.

The RF propagation analysis included creation of a baseline model from the standard off-the-shelf omnidirectional antenna, and an objective directional model for the antenna selected for testing. The objective antenna selected for this test was the Tron-Tek LS910/LP LPDA yagi antenna. Assumed conditions include: a UAV flight height of $1000 \mathrm{ft}$. above ground, $1 \mathrm{~W}$ of transmit power, $1 \mathrm{~dB}$ of systems loss, acceptable receiver sensitivity of -93 $\mathrm{dBm}, 900 \mathrm{MHz}$ ISM Frequency Band and $230 \mathrm{KHz}$ of channel bandwidth.

The baseline omni-directional antenna model was simulated [Fig. 2]. It should be noted that actual

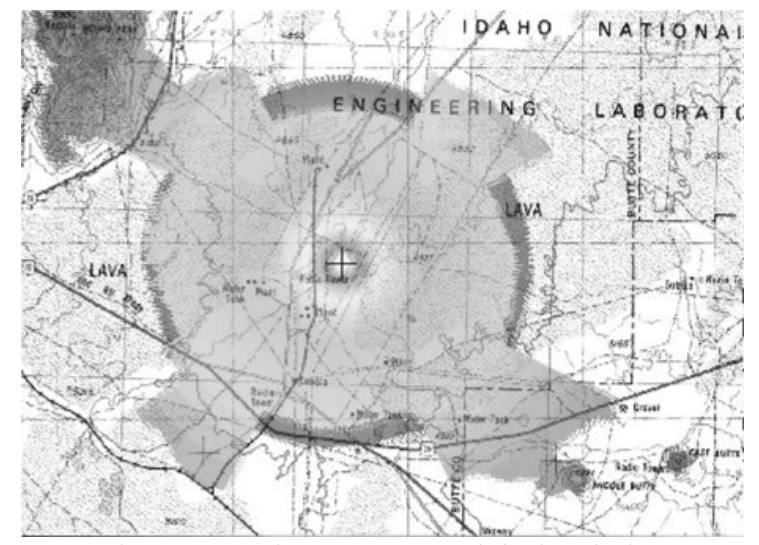

Fig. 2. Omni Antenna Model Simulation

performance in the field closely replicates the model with the exception of some interfering RF sources in the surrounding environment. Per the model maximum coverage distance using the standard omni-directional antenna at $-93 \mathrm{dBm}$ of receive power is $\sim 14.5 \mathrm{~km}$.

The objective test directional antenna model was simulated with the same conditions as the baseline configuration [Fig. 3]. Per the model maximum coverage

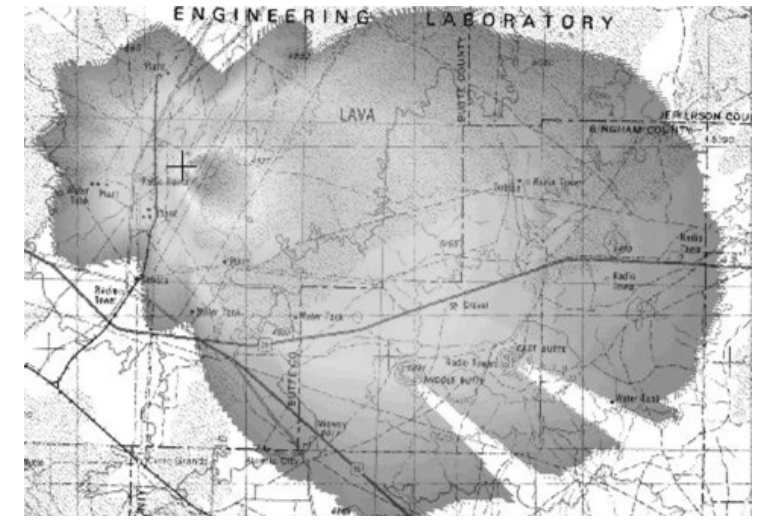

Fig. 3. Directional Antenna Model Simulation

distance using the objective directional antenna at $93 \mathrm{dBm}$ of receive power is $\sim 32.8 \mathrm{~km}$.

As would be expected, the utilization of a higher gain directional antenna versus the standard omni-directional antenna yields twice the UAV communications range. Note the range increase was a factor of antenna gain and propagation pattern and not change in receiver/transmitter configuration.

\section{DEVELOPMENT, DESIGN, AND INTEGRATION}

Integration of the dynamic tracking directional antenna was somewhat straight forward with minimal issues or materials costs. Key design considerations included: selection and mounting of the gimbaled antenna, algorithm and software design, ground station telemetry interface, and software development.

The field prototype uses a low-cost gimbaled design from Directed Perception. It is a 2-axis design that has been used with success by the INL in previous robotic efforts and it has an appropriate rate of acceleration and velocity, range of motion, and payload capacity. Future designs will include a heavier all-weather gimbal from the same manufacturer that includes continuous rotation with a microwave frequency slip ring and will have a compatible interface [Fig. 4.].

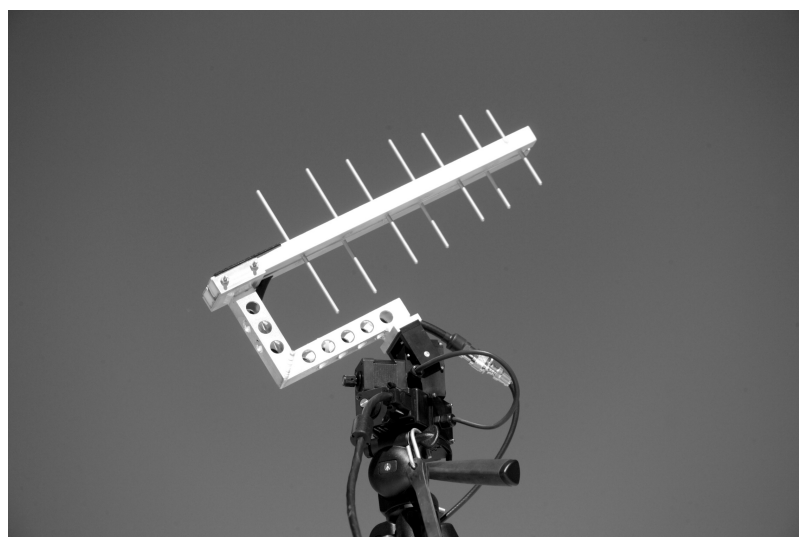

Fig. 4. Gimbaled Directional Antenna 
The interface to the ground station telemetry stream was accomplished using Cloud Cap Technology's opensource software development kit. The Windows ${ }^{\mathrm{TM}}$ Operator Interface program used to Command and Control the UAV is net workable via TCP/IP socket communications. This feature allows multiple instances of the $\mathrm{C} 2$ interface to be simultaneously active with the $\mathrm{UAV}$ as well as custom applications to be developed to seamlessly obtain mission-critical data. This capability was utilized in the development of the software interface to the 2-axis gimbal used to deploy the directional antenna.

Basic pointing algorithms were employed to determine the direction and bearing from the GPS-based location of the ground station to the GPS-based location of the airborne UAV. This data was then used to determine the necessary commands to the gimbal to target the directional antenna at the UAV to maintain communications. An application was code developed with the pointing antenna software interface actively controlling the gimbal. Field calibration and setup of the gimbaled antenna requires the system be pointed due North. Initial testing included deploying an EO zoom camera mounted to the Tron-TEK antenna to determine visually how well the UAV was being tracked by the controlling software [Fig. 5].

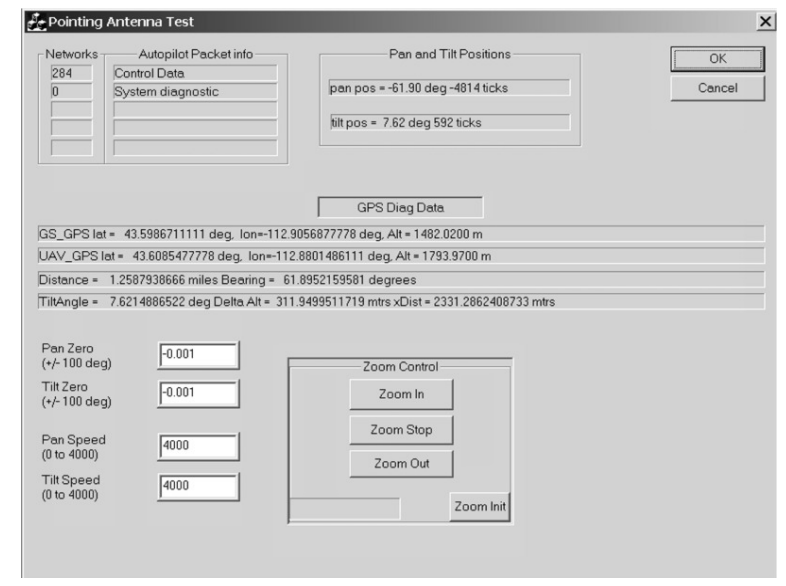

Fig. 5. Software Interface

\section{TESTING AND INITIAL RESULTS OF THE PROTOTYPE}

Initial prototype of the dynamic tracking directional antenna was deployed in September 2005. Results proved very favorable from the first set of tests. The data will be used to mature the design and support other concepts for programmatic use. It should be noted that the results at the time of writing are early in the evolution of the design. Additional tests are planned in 2005 during program spiral releases.
The September deployment was conducted in a stepwise approach. As noted in this paper, attention to details and risk management is the difference between UAV success and failure. After an extensive testing period in a controlled simulation environment, the dynamic tracking directional antenna was integrated into the system. The antenna was connected to a manual RF switch that permitted the operator to switch from the standard omnidirectional antenna to the INL gimbaled design. Should an anomalous condition be detected, the standard antenna could be returned to service without added risk to the $\mathrm{UAV}$ in autonomous or manual flight.

The other added test device included an RF signal attenuator that allowed various levels of attenuation to be added to both the baseline omni-directional antenna and the prototype dynamic tracking directional antenna. This attenuator permitted the system under test to add controlled signal loss without the need to fly the UAV at distances beyond controlled recovery areas.

With the UAV in service in a predetermined flight path, various levels of attenuation were added to the standard omni-directional antenna until a 'near' loss of communications condition was observed. This baseline test condition in effect created an environment that would resemble the UAV flying at maximum RF telemetry range. During the test this attenuation value was $-6 \mathrm{dBm}$.

Data RSSI (Received Signal Strength Indicator) levels seen at the ground station from the UAV was

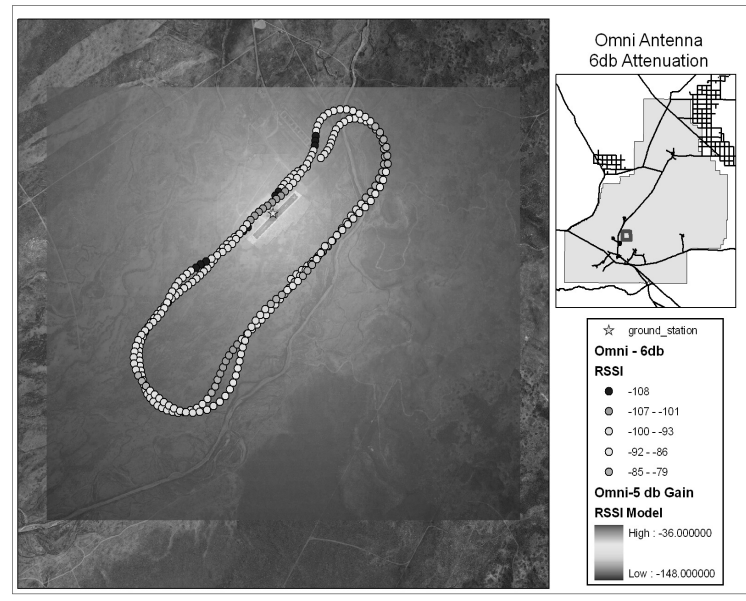

Fig. 6. Omni Antenna RSSI Profile

collected [Fig 6]. Under the same baseline conditions the dynamic tracking directional antenna was switched into the system. Data was collected with immediately observable improved results [Fig. 7.].

With the dynamic tracking direction antenna in service an immediate $6 \mathrm{dBm}$ gain in performance was observed, that more than doubling the effective range of UAV data telemetry communications from ground station to aircraft. Over the next several months, expanded tests 
will be conducted with several other selected antennas. Tests will include configuration for $\mathrm{EO} / \mathrm{Ir}$ video telemetry.

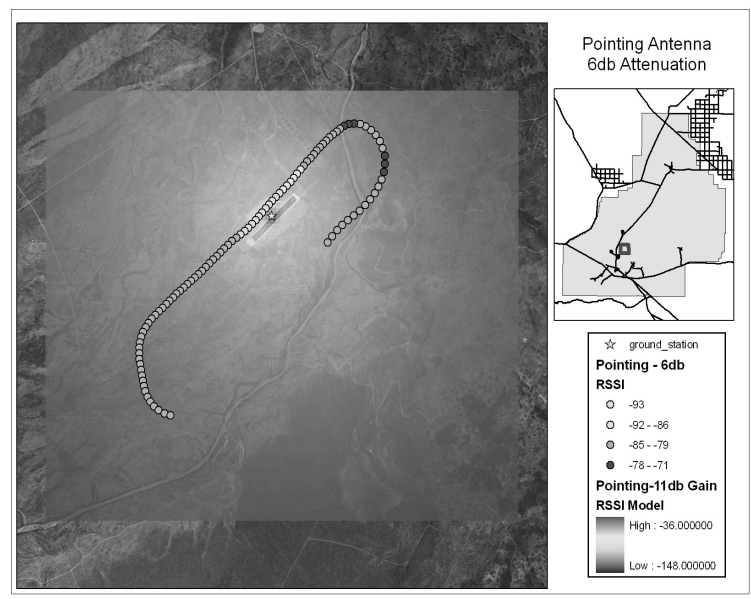

Fig. 7. Directional Antenna RSSI Profile

\section{CONCLUSIONS}

The speed at which UAVs are embraced by emergency response teams could include many factors such as: affordability, usability, sensor payloads, operations and maintenance, mission integration, reliability, and survivability. Maturity of UAV systems will occur but 'one size will never fit all' and system tools will need developed to overcome deficiencies. In the case of this effort a simple affordable dynamic tracking directional antenna improved the performance and reliability of UAV communications and therefore utilization. As missions develop, and UAV tools are put into service, other affordable technology strategies will need to be applied.

\section{ACKNOWLEDGMENTS}

We would like to acknowledge other members of the INL UAV team (Mark McKay, Jodie Boyce, Warren Jones, Lt. Robert Adolfson, and Ryan Hruska), industry partners (Cloud Cap Technologies and Arcturus USA), and the US Department of Energy (Carl Pocrasky) for interest in maturing affordable UAV technology for National and Homeland Security missions. Finally, as with all systems development, it takes a team accomplished in many areas to succeed - strength is in your partners. 